\title{
Facile conversion of tetracycline antibiotics to 4,11a-bridged derivatives via oxidative mannich cyclization
}

Jianxin Gu, Ping Cai, Yumin Gong, Mark E Ruppen and Thomas Storz

The Journal of Antibiotics (2011) 64, 167; doi:10.1038/ja.2010.157

Correction to: The Journal of Antibiotics (2010) 63, 693-698;

doi:10.1038/ja.2010.119

Due to a production error, the keywords of the article were missing. The keywords are mannich, oxidative cyclization, tetracyclines, tigecycline. 